\title{
Actualización: Intervenciones para la promoción de ambientes de trabajo saludables
}

\author{
Interventions to promote healthy work enviroments
}

Laura Luciani , Tami Guenzelovich*, Vilda Discacciati* y Sergio Terrasa* $\ddagger$

\begin{abstract}
Resumen
Basándose en los resultados de varias revisiones sistemáticas publicadas en los últimos años, este artículo describe la evidencia que sustenta las intervenciones realizadas para la promoción de ambientes de trabajo saludables. Concluye que existe moderada evidencia para respaldar la implementación de este tipo de intervenciones respecto de su eficacia para promover: 1) un aumento de la productividad y una menor tasa de ausentismo, 2) un pequeño descenso del peso y del índice de masa corporal; 2) un mayor autoreporte de consumo de vegetales y de práctica de actividad física; 4) un menor auto-reporte de estrés laboral y una mejor percepción de la propia condición física, 5) mejores parámetros lipídicos y del metabolismo de la glucemia.

Las intervenciones exitosas incluyeron actividades educativas para el desarrollo de habilidades, intervenciones comunicacionales y de comportamiento, modificaciones físicas del lugar de trabajo (cafetería, lugares y facilidades para hacer gimnasia) e intervenciones de soporte social (comisiones de empleados para planear actividades y gestionar colaciones o snacks saludables, para consensuar políticas de control tabáquico y para organizar concursos e incentivos monetarios).
\end{abstract}

\section{Abstract}

Based on the results of several systematic reviews published recently, this paper describes the evidence supporting interventions to promote healthy work environments. It concludes that there is moderate evidence to support the implementation of these interventions regarding their effectiveness in promoting: 1) an increase in productivity and a lower rate of absenteeism, 2) a small decrease in weight and body mass index, 2) greater self-reported vegetable intake and physical activity, 4) a lower self-reported work stress and a better perception of self physical condition, 5) improved lipid and glucose metabolism.

Successful interventions included educational activities for skill development, communication and behavioral interventions, physical modifications of the workplace (sanck bar, places and facilities for exercise) and social support interventions (commission integrated by employees to plan and manage activities and healthy snacks, to agree on smoking control policies and to organize competitions and monetary incentives).

Palabras clave: ambientes de trabajo, promoción de la salud. Key words: work enviroment, health promotion.

Luciani L, Guenzelovich T, Discacciati V y Terrasa S. Intervenciones para la promoción de ambientes de trabajo saludables. Evid Act Pract Ambul. Ene-Mar 2013. 16(1). 26-28.

\section{Introducción y contexto}

Un ambiente laboral saludable resulta esencial, no sólo para lograr la salud de los trabajadores, sino también para mejorar la productividad, la motivación laboral, el espíritu de trabajo, la satisfacción en el trabajo y la calidad de vida general ${ }^{1 \dagger}$. Sin embargo, pese a sus beneficios comprobados, los entornos laborales saludables no son una realidad para gran parte de la fuerza de trabajo.

La comunidad internacional, incluidas la Organización Mundial de la Salud (OMS), la Oficina Internacional del Trabajo (OIT), los sindicatos internacionales y otras entidades, ha asumido el compromiso de promover y apoyar las acciones y las medidas que tomen los países para lograr implementar lugares de trabajo saludables ${ }^{2}$. Por ejemplo, la Organización Panamericana de la Salud (OPS) considera al lugar de trabajo como un entorno prioritario para la promoción de la salud en el siglo XXl².

Por su parte, para la OPS, la estrategia de promoción de la salud en el lugar de trabajo (PSLT) incluye la realización de una serie de políticas y actividades diseñadas para ayudar a los empleadores y a los trabajadores a aumentar el control sobre su salud y a mejorarla ${ }^{2}$, favoreciendo la productividad y la competitividad de las empresas y contribuyendo al desarrollo económico y social de los países².

También en la Unión Europea este tema ha cobrado relevancia. Durante 2000 se había documentado que uno de cada tres trabajadores tenía la percepción de que estaba expuesto a algún riesgo de salud derivado de su entorno laboral . Por eso se estableció en dicha región la prioridad de mejorar la calidad del trabajo con el lema "Más y mejores trabajos" (en inglés: "More and Better Jobs") y estableciendo como expuestos a mayor riesgo a los trabajadores de la salud.
Por otro lado, en Croacia, Macedonia, Serbia y Alemania se están elaborando proyectos para evaluar y mejorar la calidad de vida de los trabajadores; mientras que otras iniciativas surgen de algunas consultoras como Great Place To Work Model, de origen norteamericano ${ }^{3}$.

Es bien sabido que muchas de las principales causas de morbimortalidad son potencialmente evitables o reducibles, lo que incluye tanto enfermedades agudas como entidades crónicas, como muchas formas de enfermedad cardiovascular y cánceres $^{5-6 \$}$. Demás está aclarar que los adultos con múltiples factores de riesgo como tabaquismo, hipertensión arterial, obesidad y sedentarismo, suelen ser empleados de alto "costo" en términos de "consumo" de servicios de salud, ausentismo, discapacidad, y escasa productividad 5 . Desde esta perspectiva de sesgo corporativo, la promoción de la salud es una inversión en "capital humano", ya que es más probable que los empleados se desarrollen mejor en su trabajo si están en las mejores condiciones de salud psico-física. También es más probable que se sientan más atraídos e interesados por su trabajo y valoren más a una compañía que los valora a ellos. En este sentido, la productividad de una compañía depende de la salud de los empleados.

\section{Un modelo teórico de abordaje}

Como se describe en la figura 1, hay varias dimensiones del entorno laboral que tienen que ver con cuán saludable es el mismo: el funcionamiento organizacional, la comunicación interna, la satisfacción individual, el balance entre el tiempo dedicado al trabajo y a la familia, y la existencia de tiempo dedicado a educación continua.

† La mayoría de los adultos pasa más de la mitad de las horas de su día en su lugar de trabajo. En este sentido, un entorno laboral saludable constituye un bien muy preciado para las personas, las comunidades y los países.

\&as diez principales causas de muerte en Estados Unidos están vinculadas de alguna manera con los comportamientos personales, particularmente hábitos que contribuyen potencialmente al desarrollo de enfermedades o a exacerbar problemas preexistentes. 
Figura 1: dimensiones que hacen al entorno laboral saludable

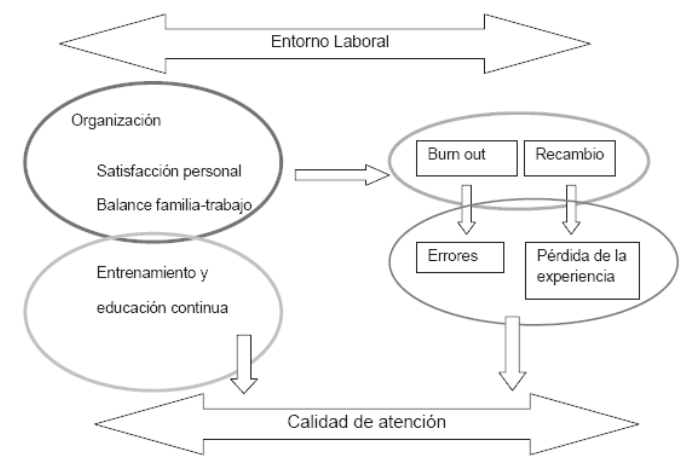

Adaptado de : Christiane Wiskow, T. A. How to create an attractive and supportive workplace. European Observatory on Health Systems and Policies (2010).

En el caso del personal de salud, la sensación de bienestar puede no sólo beneficiar al trabajador individualmente sino que también resulta esencial para brindar un servicio con mejor calidad de atención ${ }^{7}$.

\section{Los estilos de vida saludables}

Muchos estudios documentaron los beneficios en salud que conlleva la actividad física, destacándose la reducción del riesgo de enfermedad cardiovascular y de depresión, y el aumento de los niveles de satisfacción. En este sentido, en 1996 The American College of Sports Medicine y el Centers for Disease Control and Prevention (CDC) recomendaban la práctica de un mínimo de 30 minutos de actividad física de moderada intensidad la mayoría de los días de la semana, mientras que la U.S. Preventive Services Task Force recomienda actualmente incorporarla dentro de la rutina diaria ${ }^{8}$. Vale destacar también algunos resultados colectivos de un reporte de The Surgeon General basados en décadas de investigación": las personas que son usualmente inactivas pueden mejorar su salud y bienestar por el solo hecho de convertirse en moderadamente activas en forma regular; y si bien incrementando la duración, la frecuencia o la intensidad de la actividad física se obtienen mayores beneficios a nivel de la aptitud cardio-respiratoria, la actividad física no necesita ser extremadamente vigorosa para alcanzar beneficios mensurables en salud ${ }^{* *}$. Por otro lado, la actividad física regular favorecería la concentración, disminuye el estrés y cuando se la practica en grupo, sirve para afianzar lazos entre los compañeros de trabajo.

El tabaquismo es en el mundo la principal causa de muerte evitable y ocasiona alrededor de cinco millones de muertes al año. La epidemia del tabaco se está desplazando hacia los sectores pobres del mundo y actualmente el $80 \%$ de los fumadores viven en países de ingresos medios y bajos (Argentina tiene una prevalencia de tabaquismo entre las más altas de América Latina $\left.{ }^{\dagger+10,11}\right)$. En Argentina 16 de cada 100 muertes son atribuibles a esta causa y podrían evitarse si se aplicaran en forma masiva y con fuerza de Ley Federal, además de las políticas impositivas y de prohibición de la publicidad, medidas para reducir el consumo del tabaco como la restricción de fumar en espacios cerrados y la implementación de programas de cesación tabáquica en el ámbito laboral y otros ámbitos institucionales ${ }^{10}$. En cuanto a los hábitos alimentarios, es sabido que la alimentación debe ser variada para que contenga todos los nutrientes esenciales y así poder prevenir diversas enfermedades. Existe evidencia de que la reducción de la ingesta de sodio en combinación con una dieta tipo DASH ${ }^{\ddagger \ddagger}$ se asocia a una disminución de la tensión arterial'12; mientras que el estudio INTERHEART ${ }^{13}$ documentó que el $7,1 \%$ de los infartos de miocardio (IAM) en Sudamérica sería atribuible al escaso consumo de vegetales tomando como ingesta recomendada a las cinco porciones diarias. La Sociedad Española de Nutrición Comunitaria ${ }^{14}$ recomienda un consumo de alrededor de $300 \mathrm{~g}$ diarios de vegetales dia-rios $\$$; $;$ la asociación "Cinco al día", una organización intemacional destinada a promover el consumo de frutas y vegetales, propone consumir cinco raciones diarias de frutas y hortalizas frescas para mantener una dieta saludable y equilibrada.

Con respecto a los lácteos, estos aportan proteínas de elevada calidad, lactosa, vitaminas (A, D, y B12), calcio y minerales. Globalmente, se recomienda consumir dos a cuatro raciones de lácteos al día, y más aún durante el embarazo y la lactancia; recomendándose en los adultos los productos descremados, en especial en pacientes con obesidad y/o enfermedad cardiovascular. El estrés surge cuando la demanda de trabajo excede las posibilidades de controlarlo por el trabajador ${ }^{15}$ según la European Agency for Occupational Safety and Health ${ }^{16}$, pudiendo ocasionar fatiga crónica, síndrome de desgaste profesional o "burn-out", depresión, insomnio, ansiedad, cefaleas, úlceras digestivas, alergias, enfermedad cardiovascular ${ }^{17}$, músculoesqueléticas y alteraciones inmunológicas.

Respecto del impacto poblacional de los estilos de vida, mencionaremos el estudio INTERHEART ${ }^{13}$ que incluyó datos de 52 países y aportó evidencia respecto de los de mayor impacto para padecer un infarto de miocardio. Entre ellos se menciona la mala alimentación, junto a la falta de ejercicio, el consumo de tabaco y el estrés como los principales factores, que explican cerca del $60 \%$ de riesgo poblacional atribuible de infarto agudo de miocardio***.

\section{Tendencias epidemiológicas respecto del estilo de vida en Argentina}

Vale destacar las tendencias epidemiológicas de los estilos de vida en Argentina reportados por las Encuestas Nacionales de Factores de riesgo ${ }^{18}$ realizadas en Argentina durante 2005 y 2009 sobre una muestra probabilística de mayores de 18 años: 1) el segmento poblacional que reporta no realizar actividad física se incrementó desde 46,2\% (2005) hasta 54,9\% (2009); 2) aumentó la prevalencia de obesidad desde $14,6 \%$ a $18.0 \%$, manteniéndose estable la de sobrepeso $(35,4 \%) ; 3$ ) el $25,4 \%$ de la población reportó agregar siempre sal a las comidas; 4) sólo el $4,8 \%$ de la población reportó consumir diariamente al menos cinco porciones de frutas y verduras por día, evidenciándose una reducción de su consumo; 5) la prevalencia de tabaquismo activo se redujo escasamente (desde 29,7 a $27,1 \%) ; 6$ ) el reporte de exposición al humo de tabaco ajeno se redujo desde $52 \%$ a $40,4 \%$ en especial en presencia de leyes

** La actividad física reduce el riesgo de morir prematuramente, de desarrollar diabetes e hipertensión arterial y ayuda a reducir la tensión arterial en personas hipertensas; reduce los sentimientos de depresión, ansiedad, mejorando el humor; ayuda a controlar el peso y a mantener saludables los huesos, los músculos y las articulaciones (evitando dolores); ayuda a los adultos mayores a estar más fuertes y mejor adaptados a caminar y evitar caídas.

" Según una información del Ministerio de Salud de la Nación en el 2005, fumaban en la Ciudad de Buenos Aires el 34\% de los varones y el 30,1\% de las mujeres.

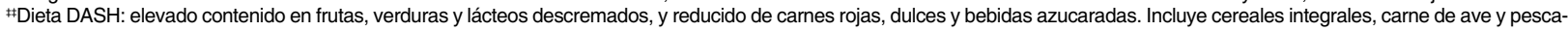
do, semillas y nueces. Mayor cantidad de potasio, calcio, magnesio, fibra y proteínas, y menor contenido de grasas y colesterol, en comparación con la dieta control.

${ }_{\$ s}$ Las frutas son indispensables porque aportan agua, azúcares, vitaminas, minerales y fibra. Se recomienda consumir tres o más frutas por día, ya que las verduras y hortalizas, fuente de vitaminas, minerales, fibras y antioxidantes.

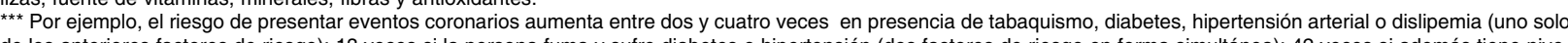
de los anteriores factores de riesgo); 13 veces si la persona fuma y sufre diabetes o hipertensión (dos factores de riesgo en forma simultánea); 42 veces si además tiene niveles lipídicos inadecuados ; 65 veces si además es obesa; y 185 veces si además reporta factores psicológicos adversos. 
provinciales $100 \%$ libres de humo.

\section{Experiencias que documentaron los beneficios de promo- cionar la salud en los ambientes laborales}

La evidencia que sustenta los efectos positivos de la promoción de la salud en el trabajo se ha desarrollado en los últimos 30 años ${ }^{19}$. Uno de los primeros ensayos clínicos en el tema ${ }^{20}$, data de 1999 y documentó los cambios de comportamiento de una cohorte de 2055 individuos que completaron tres evaluaciones en comportamientos saludables y que trabajaban en sitios que participaban en "The Working Healthy Project". Esta intervención en factores de riesgo, apuntó a promover la actividad física, la cesación tabáquica y una alimentación saludable. Fueron implementadas actividades educativas para el desarrollo de habilidades, e intervenciones comunicacionales y de soporte social y físico en el entorno de trabajo. Para tal efecto se fomentó el establecimiento de un consejo asesor de emplea-dos para planear actividades, para gestionar colaciones o snacks saludables, para consensuar políticas de control tabáquico y para organizar concursos e incentivos monetarios. Previamente a la intervención, el $38 \%$ de los participantes hacía alguna actividad física y había reportado consumir 2,7 porciones de frutas y vegetales diarias, $7,9 \mathrm{~g}$ de fibras cada 1000 calorías y $35,4 \%$ de calorías provenientes de la grasa. $28 \%$ de los participantes eran fumadores. La intervención se asoció a un incremento significativo y relevante en los niveles de actividad física, en el consumo de frutas, vegetales y fibras, pero no tuvo impacto en la cesación tabáquica ni en el consumo de grasas. Dos revisiones sistemáticas llevadas a cabo en $1995^{21}$ y $2007^{22}$ por el Centro de Control de Enfermedades de EE.UU. (CDC) concluyeron que programas bien diseñados y basados en evidencia científica, pueden contribuir a mejoras a largo plazo en la salud y en la productividad de los trabajadores ${ }^{\dagger t+}$. Además de reducir riesgos en salud y mejorar la promoción de comportamientos saludables, la Fuerza de Tareas Preventiva de los EE.UU ${ }^{19}$ comunicó que este tipo de programas se asocia a beneficios adicionales como el incremento de la conciencia del trabajador respecto de su estado de salud y mayor alerta para la detección precoz de problemas asociados a la falta de comportamientos saludables. También se realizaron experiencias en diferentes empresas con resultados financieros positivos en términos del aumento de la productividad, la disminución del ausentismo, etc. Entre ellas: Johnson and Johnson, Citibank, Bank of America y Chevron Corporation ${ }^{19}$. Un meta-análisis publicado en 2009 por Conn y col. ${ }^{23}$ resumió la evidencia respecto de las intervenciones para la promoción de la actividad física en el lugar de trabajo. Si bien los estudios primarios fueron muy heterogéneos, los revisores informaron una mejoría de la mayoría de los resultados evaluados (auto-reporte de actividad física, percepción del propio estado físico, niveles de lípidos en sangre y parámetros relacionados con el metabolismo de la glucosa, medidas antropométricas, niveles reportados de estrés laboral y atención en el trabajo). La revisión sistemática publicada por Anderson y col. ${ }^{24}$ en 2009 analizó la efectividad de programas para mejorar los hábitos alimentarios, para promover la actividad física y para lograr un peso saludable en los empleados. La mayoría de los estudios analizados habían combinado estrategias informacionales y de comportamiento sobre los trabajadores y otros también habían implementado modificaciones en el lugar de trabajo (cafetería, lugares y facilidades para hacer gimnasia) para promover elecciones saludables. Los resultados reportados entre los seis y los doce meses de las implementaciones fueron modestos, con un efecto total estimado en nueve ensayos clínicos de un descenso de 2,8 libras (IC95\% -4,6 a -1,0); y un descenso en el índice de masa corporal de $-0,5 \mathrm{~kg} / \mathrm{m}^{2}$ (IC95\% $-0,8$ a $\left.-0,2\right)$ estimado sobre la base de datos de seis ensayos clínicos.

Respecto de la costo-efectividad de este tipo de intervenciones, sobre la base de 15 estudios (ensayos clínicos aleatorizados y controlados, y estudios cuasi-experimentales) la revisión publicada en 2001 por Pelletier y col. ${ }^{25}$ analizó la costoefectividad de programas desarrollados en sitios de trabajo para la promoción de la salud y el manejo de enfermedades, concluyendo que en su mayoría los resultados fueron positivos tanto en términos de efectividad como de costos.

\section{Conclusiones}

Existe evidencia para respaldar la implementación intervenciones para la promoción de ambientes laborales saludables respecto de su eficacia para promover: 1) un aumento de la productividad y una menor tasa de ausentismo, 2) un pequeño descenso del peso y del índice de masa corporal; 2) un mayor auto-reporte de consumo de vegetales y de práctica de actividad física; 4) un menor auto-reporte de estrés laboral y una mejor percepción de la propia condición física, 5) mejores parámetros lipídicos y del metabolismo de la glucemia.

Las intervenciones exitosas incluyeron actividades educativas para el desarrollo de habilidades, intervenciones comunicacionales y de comportamiento, modificaciones físicas del lugar de trabajo (cafetería, lugares y facilidades para hacer gimnasia) e intervenciones de soporte social (comisiones de empleados para planear actividades y gestionar colaciones o snacks saludables, para consensuar políticas de control tabáquico y para organizar concursos e incentivos monetarios).

Recibido el 30/03/12 y aceptado el 15/10/12.

Referencias

1. Pencak M. Workplace heatth promotion programs. An overwiew. Nurs Clin North Am. 1991. Mar, 26 (1): $233-40$

2. Estrategia de Promoción de la Salud en Los lugares de trabajo de América Latina y el Caribe. Organización Panamericana de La Salud, Organización Mundial de la Salud. San José, Costa Rica: 2000. 3. How to create an attractive and supportive workplace. European Observatory on Healthe Systems and Ponilicies Christiane Wiskow, T. A. (2010) 4. European Foundation for the mprovement to Living and Working Conditions. ERM
5. Healthy Workforce 2010. Partnership for Prevention Washington 2001 . 5. Healthy Workforce 2010. Partnership for Prevention. Washington, 2001.
6. Actual causes of Death in The United States. Mc Ginnis Jm, Foege WH.

The Effectiveness Of I Lemaire. Physician wellness: a missing quality indicator. Lancet 2009; 374: 1714-21.

Physical Activity and Interventions To Increase Physical Activity A Systematic Review Emily B. Kahn, Phd, Mph, Leigh T. (Am J Prev Med 2002;22(4s):73-107).

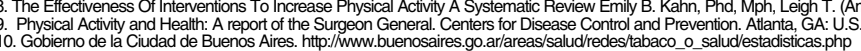

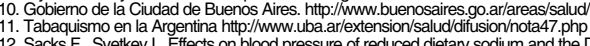

Sacks F., Svetkey L.,Effects on blood pressure of reduced dietary sodium and the Dietary Approaches to Stop Hypertension (DASH) diet. N Engl J Med 2001:344:3-10.

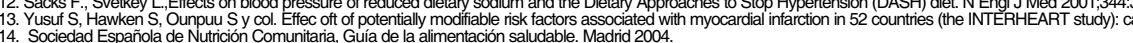

15. Kivimäki M, Leino-Arias P. Luukkonen R W Work stress and risk of cardiovascular mortality p prospective cohort study of industrial employees. BMJ 2002; $325: 857$.

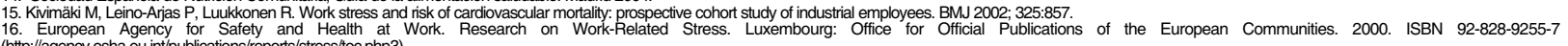

17. Rosengren A, Hawken S, Ounpuu Sy col/Association of

18. Segunda Encuesta Nacional Factores de Riesgo (ENFR) Para Enfermedades No transmisibles. Ministerio de Salud. Presidencia de la Nación. 2009.

19. Ron Z. Goetzel, phd. Workplace Health Promotion: Policy Recommendations that Encourage Employers to Support Health. Improvement Programs for their Workers 2008.

20. Emmons KM, Linnan LA, The Working Healthy Project: a worksite health -promotion trial targeting physical activity, diet, and smoking. J Jccup Environ Med. 1999. Jul 41 (7) $7545-55$.
21. Wisson MG, Holman PB, Hammock A. A comprehensive review of the effects of worksite health promotion on health-related outcomes. American Joumal of Health Promotion. 1996; 1016
22. Task Force Community Preventive Senvices. Proceedings of the Task Force Meeting: Worksite Reviews. Attanta, GA: Centers for Disease Control and Prevention, 2007.

23. Conn VS and col, Meta-analysis of workplace physical activity interventions. Am J. Prev. Med. 2009 Oct,37(4):330-9.

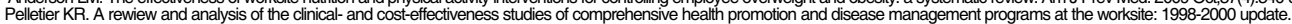

"It Por ejemplo, la revisión del CDC de 2007 examinó datos de 50 estudios que reportaban los resultados de la participación en programas basados en comportamientos saludables implementados en el sitio de trabajo y que habían incluido mediciones fisiológicas e indicadores de productividad. Si bien cuando se miden estos cambios en forma individual, las repercusiones son pequeñas, estas cobran importancia cuando se las valora en el nivel poblacional. 